\title{
Observation and Initiation of Joint Action in Infants
}

\author{
Christine Fawcett and Ulf Liszkowski \\ Max Planck Institute for Psycholinguistics
}

\begin{abstract}
Infants imitate others' individual actions, but do they also replicate others' joint activities? To examine whether observing joint action influences infants' initiation of joint action, forty-eight 18-month-old infants observed object demonstrations by 2 models acting together (joint action), 2 models acting individually (individual action), or 1 model acting alone (solitary action). Infants' behavior was examined after they were given each object. Infants in the joint action condition attempted to initiate joint action more often than infants in the other conditions, yet they were equally likely to communicate for other reasons and to imitate the demonstrated object-directed actions. The findings suggest that infants learn to replicate others' joint activity through observation, an important skill for cultural transmission of shared practices.
\end{abstract}

Action observation is a valuable learning opportunity that enables infants to acquire culturally relevant knowledge about objects and behavior from early in life (e.g., Meltzoff, 1985, 1988; Tomasello, 1999). Research on imitation has provided much evidence that infants learn individual actions through observation. However, very little is known about whether infants also learn joint activities through observation. The current study focuses on whether infants, when observing others' joint activity, encode the participants' goals to act together, such that the infants are later motivated to replicate the joint activity they observed.

Findings from research on object-directed imitation show that by 9 months of age, infants reproduce novel actions on objects, even after a delay (Herbert, Gross, \& Hayne, 2006; Meltzoff, 1988). In the 2 nd year of life, infants imitate actions with an understanding of the model's intentions: They imitate a model's intended actions more than her accidental actions (Carpenter, Akhtar, \& Tomasello, 1998), enact goals of incomplete actions (Meltzoff, 1995; Nielsen, 2009), and reproduce a model's means selectively in relation to her goal (Gergely, Bekkering, \& Király, 2002). Thus, before the age of 2 , infants can encode others' individual objectdirected goals and later replicate observed actions to achieve those goals.

Further findings reveal that 1- to 2-year-old infants' object-directed imitation is influenced by the social nature of the model. For example, infants are more likely to imitate the actions of human

Correspondence concerning this article should be addressed to Christine Fawcett, Department of Psychology, Uppsala University, 75142 Uppsala, Sweden. Electronic mail may be sent to christine.fawcett@psyk.uu.se. models than nonhuman models (e.g., mechanical pincers; Meltzoff, 1995; Slaughter \& Corbett, 2007), and to imitate the actions of a model who engages with them socially than one who acts aloof (Nielsen, 2006). Infants are also more likely to imitate a novel action when the model is present and watching them than when she has left the room or is turned away (Király, 2009), or when she is only seen on video (Nielsen, Simcock, \& Jenkins, 2008).

While these previous studies give some insight into the social factors that infants take into account when learning from observation, they focus on the goals and actions of a single model in isolation. In contrast, infants' everyday experience is a rich social environment in which they often observe several people acting together. One question is thus what infants learn when they observe interactions. Paradigms in which infants watch from a thirdperson perspective as two individuals interact have revealed that 1-year-olds differentially encode individuals' roles (Schöppner, Sodian, \& Pauen, 2006) and that 18-month-olds can learn the referent of a word (Floor \& Akhtar, 2006) or point (Gräfenhain, Behne, Carpenter, \& Tomasello, 2009), or even learn a novel action (Herold \& Akhtar, 2008) when it is presented to another person. Repacholi and Meltzoff (2007) further showed that watching others' social interactions subsequently influences 18month-olds' imitation of goal-directed actions. In their study, infants watched while an onlooker commented on the model's actions. Infants were less likely to imitate the model if she had received

(c) 2012 The Authors

Child Development (C) 2012 Society for Research in Child Development, Inc. All rights reserved. 0009-3920/2012/8302-0005

DOI: $10.1111 /$ j.1467-8624.2011.01717.x 
a negative reaction from the onlooker than if she had received a neutral reaction, suggesting that infants encode others' social relations from a thirdperson perspective and modify their own objectdirected imitation accordingly.

Another question with regard to observing interaction from a third-person perspective is whether infants also learn more specifically about the joint nature of others' activities. Joint activities can be done at different levels of "togetherness." Some simultaneous or sequential activities that appear to be done together (e.g., finding shelter under a roof, or entering a bus; see Sebanz, Bekkering, \& Knoblich, 2006) are only coordinated because the individuals have the same, unshared goals and plans. In more elaborate joint activities, coordination is a result of individuals' shared action plans which contain the individuals' intentions of acting together (Bratman, 1992), and are typically initiated and maintained through communication (Clark, 2006). In order to learn about a joint activity through observation, one must recognize both the individual goals behind others' actions and the joint goals of acting together. For example, when learning to reproduce the joint activity of soccer, it is necessary to imitate not only the individual goaldirected act of kicking the ball but to imitate the joint act of passing the ball to other players in expectation of passing the ball back and forth.

Imitation can be a tool for acquiring culturally mediated behaviors in general, and it may be especially useful in the service of learning joint activities. The critical question is whether infants attempt to replicate a joint activity after having observed it from a third-person perspective. For example, if an infant observes two people modeling a joint action on an object, will she encode their social goal of acting together in addition to their individual goals of acting on the object? If so, the infant should be motivated to reproduce the joint activity in addition to the object-directed action.

In the current study, we asked whether 18-month-old infants, when observing joint activity, recognize the social goal underlying the interaction and are motivated to reproduce the observed joint activity. As described above, by 18 months, infants can learn from third-party observations (Floor \& Akhtar, 2006; Gräfenhain et al., 2009; Herold \& Akhtar, 2008) and have an understanding of social roles (Schöppner et al., 2006). Therefore, infants should be able to recognize the social goals underlying others' actions. During the current study, infants observed the same object-directed actions demonstrated either by one model acting alone (solitary action condition), by two models acting separately (individual action condition), or by two models acting together (joint action condition). Infants were later given the chance to act on the objects themselves. To answer our primary question, we examined whether infants attempted to engage an adult (the model or their parent) to act with them on the object. If infants are indeed motivated to reproduce others' joint activities, we expected them to invite an adult to join their actions more often following observation of a joint action demonstration than following an individual or solitary action demonstration. The joint action demonstration is the only situation in which the models are acting together with a joint goal. Even though the individual action demonstration is performed by two people, they are not acting together; thus, it should not motivate infants to initiate joint action.

We also examined whether initiation of joint action was specific to an individual who was part of the original interaction (i.e., the model) by comparing how often infants' initiation attempts were directed to the model or to their parent. In addition, to ensure that any difference in initiation of joint action was not due to an overall increase in social behavior by the infant, we also examined infants' attempts to communicate with either adult for reasons other than initiating the joint activity. Finally, to test whether infants were equally motivated to replicate the object-directed actions, we examined infants' imitative actions on the object.

\section{Method}

\section{Participants}

Forty-eight 18-month-old infants (20 females; mean age $=18$ months 28 days, $S D=20$ days) participated in the study. An additional 4 infants were excluded from the analyses: 1 for experimenter error and 3 for refusing to participate. Participants were recruited from a database of parents who expressed interest in participating in research with their child. Infants were primarily white and from middle-class families, living in a medium-sized European city.

\section{Materials}

A different novel object was used in each of six trials (see Figure 1). The objects included: (a) two bicycle bells attached to a wooden base, (b) two wooden levers on a wooden base, (c) a wooden drum with two drumsticks attached to it with cords, (d) a large plastic wheel on a wooden base, 

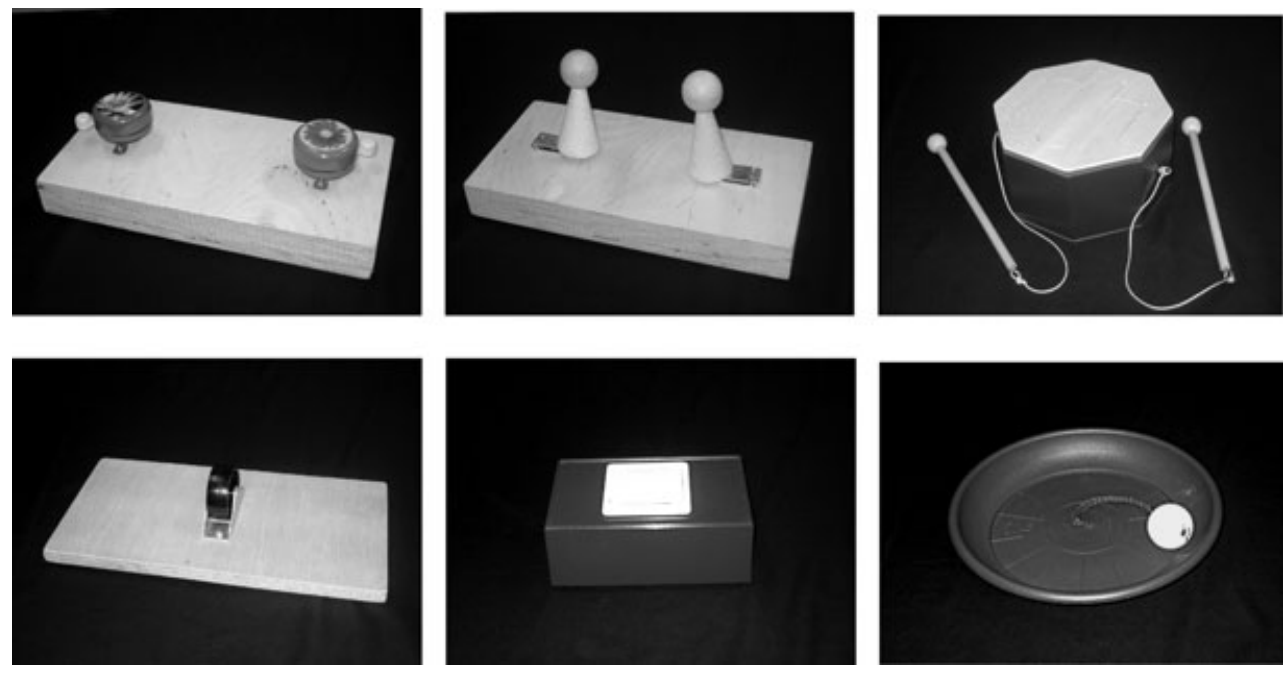

Figure 1. Objects used in each trial: bells, levers, drum, wheel, switch, trampoline.

(e) a large plastic switch on top of a plastic box, and (f) a trampoline with which a small ball could be bounced on a large plastic plate.

\section{Procedure}

Infants were randomly assigned to either the joint or solitary condition, the individual condition was run later as a control. Infants sat on their parent's lap at the head of a rectangular table with Model 1 sitting to their left and in the joint and individual action conditions, Model 2 sitting to their right (see Figure 2). Each of the six trials included a demonstration phase and a test phase. The order of presentation of objects was counterbalanced across infants.

Demonstration phase. To begin the demonstration phase, Model 1 retrieved an object from a box below the table, saying, "Oh, look at this," addressing the infant and only in the joint action condition, also addressing Model 2. In the joint action condition, the two models then took turns acting on the object and also acted on it simultaneously. Specifically, each model performed the action on the object three times in a row, then one model said, "Now together," and both models did the action simultaneously six times. Then the sequence was repeated, with each model performing the action on the object separately three times in a row, and then performing the action together six times. During each action demonstration, the model made a comment about the object (e.g., "This is fun") and made eye contact with the other model and the infant. For the objects with two manipulable parts (i.e., bells, levers, and drum), each model only acted on one part. For the objects with one manipulable part (i.e., wheel, switch, and trampoline), they both acted on the same part. Thus, the demonstration in the joint action condition allowed infants to observe that the object could be acted on by either one or two people.

In the individual action condition, the models carried out the same actions and comments as in the joint action condition. However, one model completed all of her actions and comments on the object before the other model acted on it and the models only looked at the object and the infant, never at each other. For example, Model 1 acted on the object three times using her right hand, then three times using her left hand, then six times using both hands simultaneously. While Model 1 acted on the object, Model 2 sat quietly and looked downward. Next, Model 2 acted on the object in the same way while Model 1 sat quietly and looked downward.

In the solitary action condition, Model 1 said the same statements and performed the same actions on the object as were seen in the previous conditions; however, she performed all of the actions herself using her left and right hands as in the individual action condition.

Since each action the models performed was quite brief (e.g., hitting the drum or spinning the wheel), a complete object demonstration lasted approximately $40 \mathrm{~s}$. At the end of the demonstration in the joint and individual action conditions, Model 2 excused herself from the table and waited out of view behind a screen, leaving Model 1 and the infant at the table to begin the test phase. 


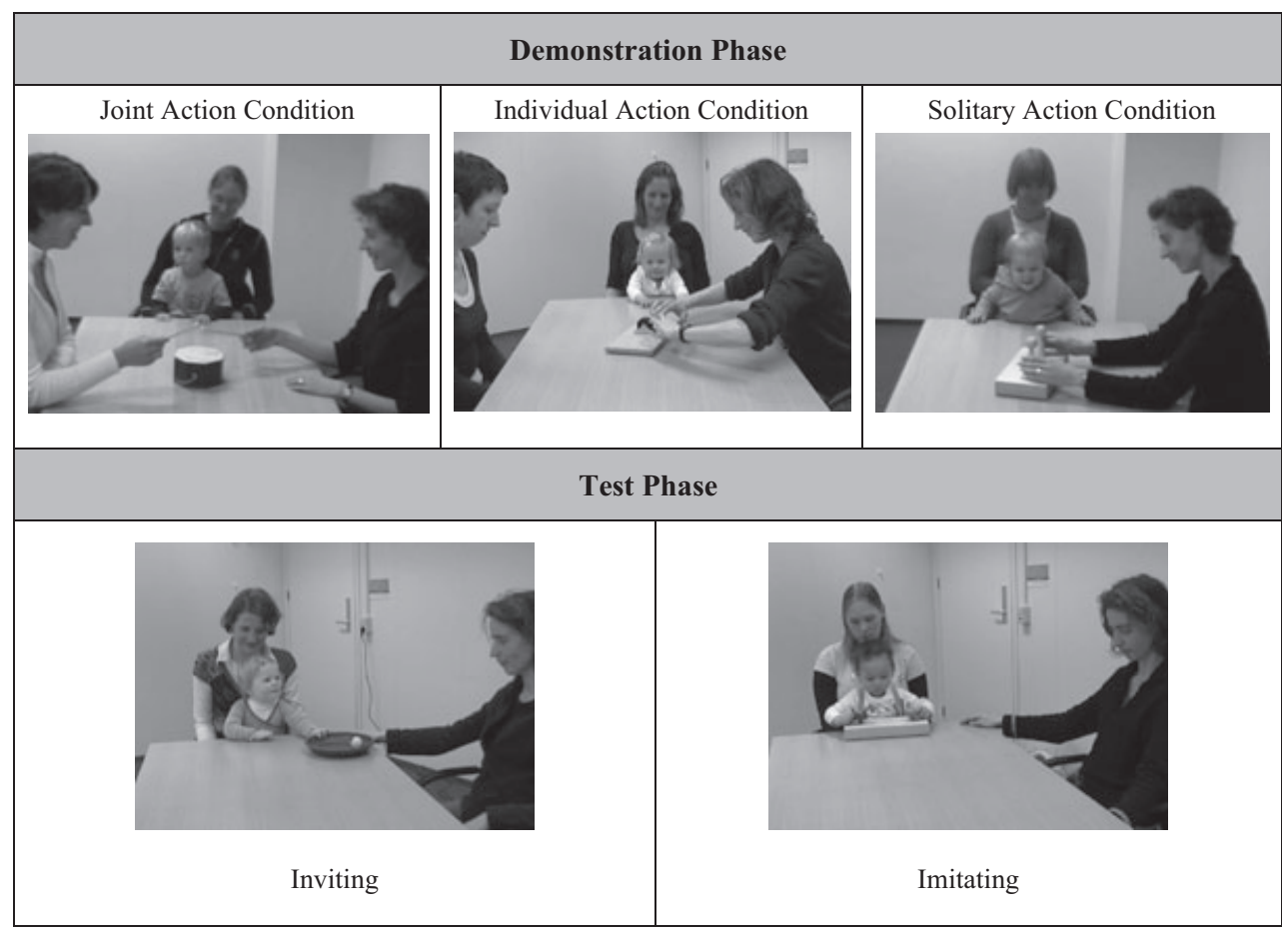

Figure 2. Demonstration phase configuration for joint, individual, and solitary action conditions.

Note. Test phase configuration with examples of inviting and imitating.

Test phase. The 90-s test phase began when Model 1 moved the object toward the infant and said, "Here, try it." She then turned to face forward, sat back in her chair and looked down at the table, with her hand remaining near the toy (see Figure 2). If an infant addressed the model, she looked at the infant, leaned slightly forward, nodded and said, "Mm hmm," and then sat back and looked down again. Parents were instructed to behave the same way if their child tried to communicate with them or give them the object. By behaving this way, the model (or parent) signaled that she was able to be interacted with, but was not initiating or reinforcing interactions with the child. At the end of the test phase in either condition, Model 1 played with the infant for a few seconds before putting the object away. This served to prevent the infant from becoming discouraged from engaging with her in subsequent trials. In the joint and individual action conditions, Model 2 returned from behind the screen to begin the next trial.

\section{Coding}

Invitations. Our main variable of interest was invitations: infants' attempts to initiate joint activity with an adult (i.e., the model or their parent). Invitations were defined as behaviors communicating that the infant wanted the adult to interact with her and the object. Each invitation included either moving the object toward the adult or moving the adult's hand to the object, as well as one or more of the following social cues: making eye contact, vocalizing, or smiling, and specifically communicating a desire for the adult to act on the object together in play. For example, an invitation to the model was coded when the infant moved the object toward the model, looked at her, and waited in expectation for her to act on the object.

Other communicative behaviors. Infants' attempts to communicate with an adult for reasons other than initiating joint activity were coded as other communicative behaviors. These behaviors occurred when the infant directed two or more social cues to the adult (i.e., moving the object toward the adult, moving the adult's hand to the object, making eye contact, vocalizing, or smiling) without attempting to initiate further joint activity. For example, if an infant made eye contact and smiled at the model while lifting up the object, as with a showing gesture (Bates, Camaioni, \& Volterra, 1975), her behavior did not convey a clear expectation that the model should act on the object and it could simply communicate that the infant wanted to show the model the object. As a second example, if an infant moved the object toward the model and 
vocalized to her, but then disengaged from the object, it could communicate that the infant wanted to get rid of the object. In sum, unless a communicative attempt showed a clear expectation from the infant that she desired the adult to join in acting on the object, the behavior was coded as other communicative behavior rather than invitation.

Object-directed imitation. When infants performed the demonstrated actions on the object (e.g., ringing the bells on the bell object), the duration of the behavior was coded as imitation.

Reliability. In addition to the main coder who was blind to the study's hypotheses, a second coder coded four randomly selected infants in each condition $(25 \%)$ to assess reliability. Intraclass correlations (ICC; Shrout \& Fleiss, 1979) revealed significant agreement between the coders on the number of invitations (ICC $=.79, p<.001$ ), the number of other communicative behaviors (ICC $=.92, p<.001$ ), and the duration of imitation per infant (ICC $=.92$, $p<.001)$. For object-directed imitation, coders also agreed on whether imitation occurred in a trial in all but one case (99\% agreement, 72 cases).

\section{Results}

Preliminary analyses revealed no gender differences on any of the reported measures. A total of 11 trials were removed from the analyses due to children's unwillingness to play ( $n=7$ trials across 4 infants), parental interference ( $n=1$ trial), or experimenter error ( $n=3$ trials across 3 infants).

Our primary hypothesis was that infants in the joint action condition would be more likely to invite an adult to join their activity than those in the individual or solitary action conditions. Infants' number of invitations per trial was analyzed with a mixed-effect Poisson regression model. The Poisson distribution was used since it is appropriate for count data. The model was fit using the lmer function of the R package lme4 (Bates, 2005; Bates \& Sarkar, 2007; R Development Core Team, 2005). The model included condition and person (model or parent) as fixed factors with the joint action condition as the baseline against which the individual and solitary action conditions were compared. Random effects for subjects and objects were also included to account for any nonindependence among infants' responses and among the responses to the different objects (Baayen, Davidson, \& Bates, 2008). As predicted, the results showed that infants made more invitations in the joint action condition than either the individual $(\beta=-1.31, z=-2.20$, $p=.03)$ or solitary $(\beta=-1.03, z=-2.01, p=.04)$ action conditions, suggesting that infants were motivated to reproduce the joint activity they observed (see Figure 3). We also tested for learning or fatigue effects over the trials and found no differences between the rate of inviting for Trials 1 and 2 compared to Trials 5 and $6, t(47)=-1.50$, $p=.14$.

A secondary question was whether infants' invitations would be directed to both the model and their parent equally. The results showed that infants were overall more likely to direct invitations to the model than to their parent $(\beta=1.27, z=2.05$, $p=.04)$. There was a trend for an interaction between person and condition $(\beta=1.48, z=1.81$, $p=.07)$, suggesting that this effect was not present in the individual action condition in which infants were equally likely to direct invitations to the model or their parent.

To ensure that any difference in initiation of joint action was not due to an overall increase in social behavior by the infant, we examined infants' attempts to communicate with either adult for reasons other than initiating the joint activity. A possible alternative explanation for infants' higher rate of inviting is that the joint action condition motivated infants to engage socially overall, rather than specifically for the purpose of initiating joint activity. If so, infants would also show more other communicative behaviors in the joint action condition than in the other two conditions. We analyzed the

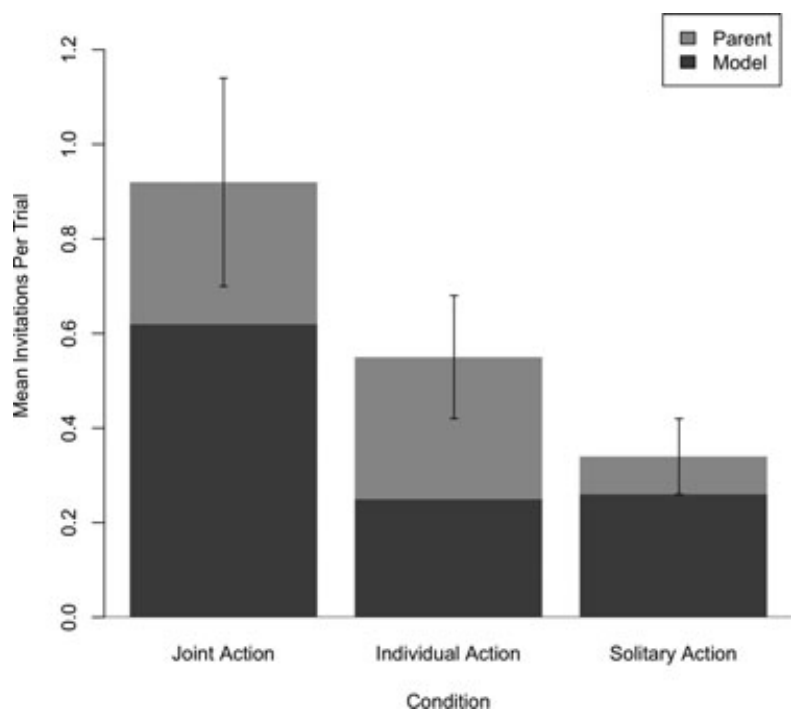

Figure 3. Mean number of invitations per trial to the model and parent in the joint, individual, and solitary action conditions. Note. Bars indicate standard errors. 
number of other communicative behaviors in the same way as the number of invitations. The analysis revealed that there were no significant differences between conditions. If anything, we found only a marginally significant trend in the opposite direction with more communicative behaviors in the individual than the joint action condition ( $\beta=0.60, z=1.82, p=.07)$. Thus, infants' motivation to socially engage an adult for reasons other than inviting was not greater in the joint action condition and further suggests that the influence of the joint action demonstration was specific to initiating joint activity.

Finally, to examine whether infants were equally motivated to replicate the object-directed actions they observed in the demonstration, we compared their object-directed imitation across the three conditions. All infants performed the demonstrated actions at least once, with no significant difference across the conditions in the proportion of trials with imitation (joint $M=0.93$, individual $M=0.97$, solitary $M=0.94), F(2,45)=0.71, p=.50$, or in the amount of time infants spent imitating per trial (joint $M=36.88 \mathrm{~s}$, individual $M=37.80 \mathrm{~s}$, solitary $M=39.67 \mathrm{~s}), F(2,45)=0.38, p=.69$. Thus, infants in all conditions attended to the demonstrations, learned what action could be performed on each object, and replicated that action. As an additional control, the data on invitations and other engagement attempts were reanalyzed using only the trials in which infants imitated the object-directed actions, and we obtained the same results as described above.

\section{Discussion}

Infants who observed two models acting together on an object not only imitated their object-directed actions, but also attempted to reproduce their joint activity as a whole, as evidenced by their inviting of an adult to join their action. This finding suggests that when observing joint activities, 18-month-old infants encode others' individual object-directed goals and joint social goals. In addition, infants adopt these goals, motivating them to replicate both object-directed actions and joint activities.

Infants' motivation to invite the adult to engage in joint activity was not based on an overall increased social motivation in the joint action condition. First, the model(s) behaved equally socially with infants in all conditions, making it unlikely that infants saw the model as more socially engaging or friendly in the joint action condition. Second, the results showed that infants did not engage in more of the other communicative behaviors in the joint action condition. Thus, infants were not reluctant to approach an adult in the individual or solitary action conditions. Instead, the difference in inviting across conditions appears to be based specifically on a motivation to replicate the joint activity they observed.

Importantly, the individual action condition gives further support that infants recognize the joint goal present in the joint action condition. That is, simply seeing two people act on the toy is not sufficient to motivate infants to engage another in joint play. Only in the case where the models were truly acting together did infants also show a desire to act together as well.

The results of the current study suggest that infants' motivation to replicate a joint activity is not necessarily limited to the person who modeled it. Infants also invited their parent to act on the object. While we found that infants invited the model significantly more often than their parent overall, this difference could simply be an effect of the experimental setup in which it was easier to move the object to the model (who sat next to them at the table) than to their parent (on whose lap they sat). Thus, infants may be just as likely to invite someone other than the model if it is equally easy to do so. An additional finding was a marginally significant interaction regarding invitations to parents, such that for the individual action condition, the proportion of invitations to the parent and model were equal, while in the other two conditions, the model received more invitations than the parent. It is not clear what the source of this effect is and it is certainly an interesting issue for future research to examine the effects of different social demonstrations on infants' interactions with parents and others.

Another issue is whether infants' motivation to initiate joint action would extend to objects that were not demonstrated in the joint activity. It is currently unclear whether infants learned in the demonstration that the specific object is for joint activity or rather that the models had a more general social goal of acting together. We believe that if either the demonstrated object or the original model is present at test, infants would see the joint action demonstration as relevant and be motivated to replicate the joint activity.

Eighteen-month-old infants were chosen for the current study because previous studies have demonstrated third-party learning and imitation (e.g., Floor \& Akhtar, 2006; Gräfenhain et al., 2009; Herold \& Akhtar, 2008) as well as synchronic imitation in 
the course of social interactions (Asendorpf, Warkentin, \& Baudonnière, 1996; Nielsen \& Dissanayake, 2004) at this age. It is an empirical question whether even younger infants could recognize and attempt to reproduce joint goals in others' interactions. One possibility is that the ability depends in part on infants' own participation in social interactions with joint goals and that only after some direct experience of acting together with others do infants recognize as an observer when joint goals are underlying others' interactions. In addition, theories of the development of intentional understanding suggest that a more abstract third-person understanding of intention emerges around 1824 months, when infants are becoming more skilled in differentiating self from other (Barresi \& Moore, 1996).

Our findings also have implications for other research on joint action and cooperation that use demonstrations by one (e.g., Brownell, Ramani, \& Zerwas, 2006) or two (e.g., Warneken, Chen, \& Tomasello, 2006) individuals. Based on the current findings, having two individuals demonstrate a task together will increase children's motivation to engage with another, while having only one individual demonstrate could suppress this motivation. Thus, it is an issue for researchers to consider when designing future experiments.

Overall the current study is an important step into exploring how young children understand and are influenced by actions they observe. Our results show that seeing others perform an activity together leads infants to want to perform this activity together as well. While much previous research has shown that infants learn about others' individual actions through observation, the current findings are the first to demonstrate that infants also learn about others' joint activities through observation. This type of social learning provides a powerful way of acquiring shared practices. The current research thus extends cultural learning accounts by revealing new aspects of infants' sophisticated social cognitive skills that provide a basis for cultural creation and participation.

\section{References}

Asendorpf, J. B., Warkentin, V., \& Baudonnière, P.-M. (1996). Self-awareness and other-awareness II: Mirror self-recognition, social contingency awareness, and synchronic imitation. Developmental Psychology, 32, 313-321.

Baayen, R. H., Davidson, D. J., \& Bates, D. M. (2008). Mixed-effects modeling with crossed random effects for subjects and items. Journal of Memory and Language, $59,390-412$.

Barresi, J., \& Moore, C. (1996). Intentional relations and social understanding. Behavioral and Brain Sciences, 19, 107-122.

Bates, D. (2005). Fitting linear models in R using the lme4 package. R News, 5, 27-30.

Bates, E., Camaioni, L., \& Volterra, V. (1975). The acquisition of performatives prior to speech. Merrill-Palmer Quarterly, 21, 205-226.

Bates, D., \& Sarkar, D. (2007). Ime4: Linear mixed-effects models using S4 classes. R package, version 0.9975-13.

Bratman, M. E. (1992). Shared cooperative activity. The Philosophical Review, 101, 327-341.

Brownell, C. A., Ramani, G. B., \& Zerwas, S. (2006). Becoming a social partner with peers: Cooperation and social understanding in one-and two-year-olds. Child Development, 77, 803-821.

Carpenter, M., Akhtar, N., \& Tomasello, M. (1998). Fourteen-through 18-month-old infants differentially imitate intentional and accidental actions. Infant Behavior and Development, 21, 315-330.

Clark, H. H. (2006). Social actions, social commitments. In N. J. Enfield \& S. C. Levinson (Eds.), The roots of human sociality: Culture, cognition, and interaction (pp. 126-150). Oxford, UK: Berg.

Floor, P., \& Akhtar, N. (2006). Can 18-month-old infants learn words by listening in on conversations? Infancy, 9, 327-339.

Gergely, G., Bekkering, H., \& Király, I. (2002). Developmental psychology: Rational imitation in preverbal infants. Nature, 415, 755.

Gräfenhain, M., Behne, T., Carpenter, M., \& Tomasello, M. (2009). One-year-olds' understanding of nonverbal gestures directed to a third person. Cognitive Development, 24, 23-33.

Herbert, J., Gross, J., \& Hayne, H. (2006). Age-related changes in deferred imitation between 6 and 9 months of age. Infant Behavior \& Development, 29, 136-139.

Herold, K. H., \& Akhtar, N. (2008). Imitative learning from a third-party interaction: Relations with self-recognition and perspective taking. Journal of Experimental Child Psychology, 101, 114-123.

Király, I. (2009). The effect of the model's presence and of negative evidence on infants' selective imitation. Journal of Experimental Child Psychology, 102, 14-25.

Meltzoff, A. N. (1985). Immediate and deferred imitation in fourteen-and twenty-four-month-old infants. Child Development, 56, 62-72.

Meltzoff, A. N. (1988). Infant imitation after a 1-week delay: Long-term memory for novel acts and multiple stimuli. Developmental Psychology, 24, 470-476.

Meltzoff, A. N. (1995). Understanding the intentions of others: Re-enactment of intended acts by 18 -month-old children. Developmental Psychology, 31, 838-850.

Nielsen, M. (2006). Copying actions and copying outcomes: Social learning through the second year. Developmental Psychology, 42, 555-565. 
Nielsen, M. (2009). 12-month-olds produce others' intended but unfulfilled acts. Infancy, 14, 377-389.

Nielsen, M., \& Dissanayake, C. (2004). Imitation, pretend play and mirror self-recognition: A longitudinal investigation through the second year. Infant Behavior and Development, 27, 342-365.

Nielsen, M., Simcock, G., \& Jenkins, L. (2008). The effect of social engagement on 24-month-olds' imitation from live and televised models. Developmental Science, 11, 722-731.

R Development Core Team. (2005). R: A language and environment for statistical computing. Vienna, Austria: Foundation for Statistical Computing.

Repacholi, B. M., \& Meltzoff, A. N. (2007). Emotional eavesdropping: Infants selectively respond to indirect emotional signals. Child Development, 78, 503.

Schöppner, B., Sodian, B., \& Pauen, S. (2006). Encoding action roles in meaningful social interaction in the first year of life. Infancy, 9, 289-311.
Sebanz, N., Bekkering, H., \& Knoblich, G. (2006). Joint action: Bodies and minds moving together. Trends in Cognitive Sciences, 10, 70-76.

Shrout, P. E., \& Fleiss, J. L. (1979). Intraclass correlations: Uses in assessing rater reliability. Psychological Bulletin, $86,420-428$.

Slaughter, V., \& Corbett, D. (2007). Differential copying of human and nonhuman models at 12 and 18 months of age. European Journal of Developmental Psychology, 4, 31-45.

Tomasello, M. (1999). Social cognition before the revolution. In P. Rochat (Ed.), Early social cognition: Understanding others in the first months of life, (pp. 301-314). Mahwah, NJ: Erlbaum.

Warneken, F., Chen, F., \& Tomasello, M. (2006). Cooperative activities in young children and chimpanzees. Child Development, 77, 640-663. 